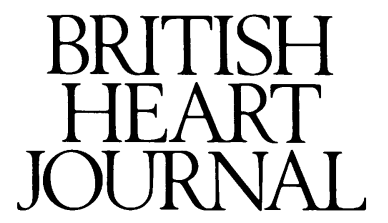

\title{
Editorial
}

\section{Magnesium and the myocardium}

The publication of the results of the Leicester Intravenous Magnesium Intervention Trial (LIMIT-2) produced headlines in the national press. There was a $24 \%$ relative reduction in mortality and a $25 \%$ reduction in the incidence of heart failure, benefits conferred equally on thrombolysed and non-thrombolysed patients.' Magnesium is clearly here to stay, but little is known of this fascinating cation despite its presence at the centre of the chlorophyll molecule and as part of the active binding site of ATPase. In humans magnesium is a physiological necessity, but it is also active pharmacologically. This has resulted in considerable confusion-particularly in the area of cardiac arrhythmia.

\section{Cardiovascular consequences of magnesium depletion}

In a classic description of magnesium deficiency, Whang and Welt described cerebral irritability with muscle tetany and ataxia in dogs. ${ }^{2}$ Rapidly progressive hypomagnesaemia was associated with severe hypokalaemia that was resistant to treatment with potassium supplementation. Histologically there was calcification of the myocardium with pathological arterial fibrinoid changes. A progressive cardiomyopathy was seen with a rise in intracellular calcium and mitochondrial calcium deposition. ${ }^{3}$

In humans there was a similar picture of calcium overload associated with myocardial magnesium depletion in cardiac transplant recipients rendered (incidentally) magnesium deficient by cyclosporin. ${ }^{4}$ Cardiac arrhythmia was not seen. In postmortem studies in ischaemic heart disease, myocardial magnesium content was up to $20 \%$ lower and calcium content up to six times higher than in non-ischaemic controls, particularly when death was sudden. ${ }^{5}$ Myocardial potassium content was concurrently reduced. Hypomagnesaemia is an independent risk factor for patients with heart failure but it is not associated with an increased incidence of arrhythmia on Holter monitoring. Lipid profiles are adversely affected with a reduction in the HDL/LDL ratio contributing to the adverse cardiovascular profile of magnesium deficiency.

\footnotetext{
Magnesium, potassium, and cardiac arrhythmia Myocardial magnesium depletion is associated with important intracellular potassium deficiency. Cardiac arrhythmia is well described in patients with hypomagnesaemia or myocardial magnesium depletion, or both, but in all reports magnesium imbalance was associated with concurrent hypokalaemia. This association precludes the identification of magnesium depletion as the single cause of arrhythmia. The myth surrounding magnesium depletion and cardiac arrhythmia is seen to arise from the dubious deduction that because intravenous magnesium abolishes
}

arrhythmia in hypomagnesaemic subjects, hypomagnesaemia is therefore the cause of the arrhythmia. However, magnesium modifies ventricular tachycardia and other arrhythmias, irrespective of serum magnesium concentration, in many patients who have no reason to be magnesium deficient. Cardiac arrhythmia was not seen in the animal studies of severe magnesium depletion.

\section{Magnesium as an antiarrhythmic agent}

In $1935 \mathrm{Zwillinger} \mathrm{used} \mathrm{intravenous} \mathrm{magnesium} \mathrm{to} \mathrm{treat}$ ventricular arrhythmia associated with digoxin toxicity. ${ }^{6}$ Since then there have been numerous case reports of the efficacy of magnesium in both supraventricular and ventricular tachycardia, albeit with an unfortunate dearth of randomised, controlled trials. Since the Cardiac Arrhythmia Suppression Trial, the benefits of a safe, cheap, "natural" drug are more readily appreciated and the use of magnesium as an antiarrhythmic agent is once again becoming popular.

Five to ten mmol of magnesium sulphate administered over a few minutes will abolish torsade de pointes (by an effect on phase 4 calcium current), reduce digoxinassociated arrhythmia (by modification of membrane digoxin kinetics), and terminate (or render pace-terminable) many sustained ventricular tachycardias. It is salutary to note that in many studies, magnesium was effective in restoring sinus rhythm when used as a "last resort" after the prior administration of a mixture of other agents.

Patch-clamp studies showed that the magnesium ion modifies at least three potassium channels, as well as acting on both $\mathrm{L}$ and $\mathrm{T}$ type calcium channels. ${ }^{7}$ The documented electrophysiological effects of magnesium are largely confined to slowing of conduction in the atrioventricular node, but recent evidence from the denervated heart in vivo suggests that prolongation of the action potential does occurr (as predicted from in vitro studies). An increase in tachycardia cycle length was seen in clinical practice. ${ }^{8}$ These observations strongly suggest a mode of action for magnesium as an antiarrhythmic agent and should begin to dispel the scepticism surrounding its use.

\section{Magnesium as a calcium antagonist}

Magnesium has been dubbed "nature's calcium antagonist", an effect most clearly seen in the antagonistic roles of magnesium and calcium in the determination of arterial tone. The action of endothelium relaxing factor (EDRF) is magnesium dependent and is antagonised by calcium. Spasm in angiographically normal coronary arteries was lessened or abolished by an intravenous bolus of magnesium, and there was some suggestion that hypomagnesaemia can provoke spasm. ${ }^{9}$

The haemodynamic effects of magnesium in vivo are 
largely mediated by a fall in systemic vascular resistance secondary to arteriolar vasodilatation. An intravenous bolus sufficient to double serum concentrations is cleared from the blood within 20 minutes, largely by renal excretion. Optimistic trials exploiting these effects to treat systemic hypertension with oral magnesium have met with little success-rapid clearance by the kidney prevents serum concentrations rising above normal. ${ }^{10}$

The use of intravenous magnesium in the acute treatment of uncontrolled pregnancy related hypertension (preeclampsia) is established in Australia and the United States, but for some reason has not proved popular in Europe. The intense vasoconstriction associated with this condition is particularly sensitive to the dilatatory effects of magnesium, which also acts in neurological tissue as a membrane stabiliser to reduce muscular and cerebral irritability. Indeed, the first use of magnesium in humans related to an attempt to induce "anaesthesia" in two intrepid researchers, who discovered that the illusion of anaesthesia was in fact a state of profound muscle relaxation bounding on paralysis! ${ }^{11}$

\section{Magnesium as an anti-ischaemic agent}

Meta-analysis of several trials has suggested a beneficial role for intravenous magnesium infused in the 24 hours after acute myocardial infarction. ${ }^{12}$ The most recent of the International Studies of Infarct Survival (ISIS 4), investigating the effects of oral nitrates and angiotensin converting enzyme inhibitors includes a similar period of intravenous magnesium administration in a $2 \times 2 \times 2$ factorial design, and will complement the results of the LIMIT-2 study.

The mechanisms of action of magnesium in this circumstance are unknown. A significant reduction in the incidence of cardiogenic shock was noted in one study, which, taken with the LIMIT-2 data, suggests that magnesium may preserve left ventricular function by reducing ischaemic damage. Evidence from animal models suggests that this may be due to reduction in calcium-mediated ischaemic damage. During ischaemia, intracellular free magnesium concentrations rise rapidly as the formation of ATP from ADP (incorporating magnesium) is limited by hypoxia and acidosis with subsequent loss of magnesium from the cell magnified by cAMP mediated $\beta$ receptor stimulation. ${ }^{13}$ During reperfusion, magnesium is re-incorporated into ATP to produce a net fall in intracellular free magnesium with reciprocal calcium overload.

In addition to any possible antiarrhythmic action, magnesium raises fibrillation thresholds; it also has antiplatelet effects mediated via prostacyclin, prolonging bleeding time. ${ }^{14}$ The beneficial effects of magnesium on arterial tone may also be important in what will prove to be a very complex relation.

Magnesium deficiency is probably an important risk factor in cardiovascular disease that has adverse effects on arterial tone and myocardial calcium balance. The arrhythmogenic effects of hypomagnesaemia are not seen in the absence of concomitant potassium imbalance. Therapeutically, magnesium has many of the characteristics of the ideal drug - it is simple, cheap, has a wide therapeutic range, a short half-life and a limited capacity for adverse interaction with other agents. As an adjuvant antiarrhythmic agent for the acute control of ventricular tachycardia, magnesium might be considered as a first line agent. Its potential anti-ischaemic actions are becoming established in myocardial infarction, but it might also prove useful in unstable angina and in the peri and post angioplasty periods.

T A MILLANE A J CAMM

Department of Cardiological Sciences,

St George's Hospital Medical School,

London

1 Woods K, Fletcher S, Roffe C, Haider Y. Intravenous magnesium sulphate in suspected myocardial infarction: results of the second Leicester Intravenous Magnesium Intervention Trial (LIMIT-2). Lancet 1992;339. 1553-8.

2 Whang $\mathbf{R}$, Welt $\mathbf{L}$. Observations in experimental magnesium depletion. I Clin Invest 1963;42:305-13.

3 Heggtveit $H$. The cardiomyopathy of magnesium-deficiency. In: Bajusz $E$, ed. Electrolytes and cardiovascular diseases. Basel/New York: S Karger, 1965:204-20.

4 Millane T, Jennison S, Patel M, Moss R, Holt D, McKenna W. Progressive myocardial calcification associated with prolonged hypomagnesemia afte cardiac transplantation [abstr]. J Am Coll Cardiol 1992;19:396.

5 Johnson CJ, Peterson DR, Smith EK. Myocardial tissue concentrations of magnesium and potassium in men dying suddenly from ischaemic heart disease. Am J Clin Nutr 1979;32:967-70.

6 Zwillinger L. Uber die Magnesiumwirkung auf das Herz. Klin Wochnenschr 1935;14:1429-33.

7 Agus Z, Morad M. Modulations of cardiac ion channels by magnesium. Annu Rev Physiol 1991;53:299-307.

8 DiCarlo LA, Morady F, de Buitleir M, Krol RB, Schurig L, Annesley TM. Effects of magnesium sulfate on cardiac conduction and refractoriness in humans. J Am Coll Cardiol 1986;7:1356-62.

9 Miyagi H, Yasue H, Okumura K, Ogawa H, Goto K, Oshima S. Effect of magnesium on an anginal attack induced by hyperventilation in patients magnesium on an anginal attack induced by hype

10 Cappuccio FP, Markandu ND, Beynon GW, Shore AC, Sampson B, MacGregor GA. Lack of effect of oral magnesium on high blood pressure: a double blind study. Br Med J 1985;291:235-8.

11 Neuwirth I, Wallace G. On the use of magnesium as an aid to anaesthesia. $J$ Pharmacol Exp Ther 1929;35:171-87.

12 Teo K, Yusuf S, Collins R, Held P, Peto R. Effects of intravenous magnesium in suspected myocardial infarction: overview of randomised trials. $B$ Med $J$ 1991;303:1499-504.

13 Romani A, Scarpa A. Hormonal control of $\mathrm{Mg}$ transport in the heart. Nature 1990;346:841-4.

14 Lauler DP, Seelig M, Hollifield JW, et al. A symposium: Magnesium deficiency-pathogenesis, prevalence, and strategies for repletion. Am J deficiency-pathogenesis 